\title{
Interface structures of InGaAs/InGaAsP/InGaP quantum well laser diodes grown by metalorganic chemical vapor deposition on GaAs substrates
}

\author{
A. Bhattacharya and L. J. Mawst ${ }^{\mathrm{a})}$ \\ Reed Center for Photonics, Department of Electrical Engineering, University of Wisconsin-Madison, \\ Madison, Wisconsin 53706 \\ S. Nayak \\ Materials Science Program, University of Wisconsin-Madison, Madison, Wisconsin 53706 \\ J. Li and T. F. Kuech \\ Department of Chemical Engineering, University of Wisconsin-Madison, Madison, Wisconsin 53706
}

(Received 27 October 1995; accepted for publication 12 February 1996)

\begin{abstract}
We have studied the effects of substrate misorientation on the growth of strained-layer $\mathrm{In}_{0.18} \mathrm{Ga}_{0.82} \mathrm{As}$ quantum well laser structures with InGaAsP confinement layers and $\mathrm{In}_{0.5} \mathrm{Ga}_{0.5} \mathrm{P}$ cladding layers lattice matched to a GaAs substrate. Low-temperature photoluminescence (PL) and atomic force microscopy (AFM) provide evidence of a strong substrate-orientation dependence of the interface structure. The surface morphology of the InGaAs quantum well is found to be determined primarily by the underlying InGaAsP confinement layer. Structures grown on exact-(100) oriented substrates exhibit three-dimensional island surface morphology, whereas growths on (100) substrates oriented $2^{\circ}$ towards [110] exhibit high surface roughness, possibly due to step bunching. These observations correlate well with previously reported device performance from strained quantum well laser diodes in the InGaAs/InGaAsP/InGaP material system, and can serve as a tool to optimize device performance. (C) 1996 American Institute of Physics. [S0003-6951(96)03716-0]
\end{abstract}

The aluminum-free InGaAs/InGaAsP/InGaP material system is increasingly important for the fabrication of semiconductor lasers. The advantages of using the Al-free system for laser diodes over the conventional AlGaAs-based material system are: (1) low surface recombination ${ }^{1}$ in this material system results in lower facet temperatures in laser diodes, permitting reliable operation at high output powers, (2) higher electrical, ${ }^{2}$ and thermal ${ }^{3}$ conductivity of InGaP cladding layers results in better total power-conversion efficiencies at high powers, and (3) novel index-guided structures can be easily fabricated due to the absence of aluminum containing compounds at the regrowth interface. ${ }^{4}$ Optimization of such devices however requires an understanding of the nature of quantum well $(\mathrm{QW})$ growth in this material system. Recently, studies relating interface structure to photoluminescence (PL) spectra have been carried out for quantum well AlGaAs/GaAs, ${ }^{5}$ strained InGaAs/GaAs (Ref. 6) and InGaAs/InP (Ref. 7) structures. Interface studies by atomic force microscopy (AFM) on MOVPE-grown (In,Ga)(As,P) materials, have mainly focused on growth mechanisms; few studies have correlated interface structure with device performance. Furthermore, the interfacial structure of strainedlayer InGaAs/InGaAsP QW structures has not been reported.

Al-free lasers with GaAs or low-band-gap InGaAsP confinement layers exhibit strong carrier leakage from the InGaAs QW to the optical confinement layers, resulting in a strong temperature dependence of both threshold current and differential quantum efficiency. ${ }^{8-10}$ To minimize this problem, higher band-gap InGaAsP confinement layers and mul-

${ }^{a)}$ Electronic mail: mawst@engr.wisc.edu tiple quantum wells are used to reduce carrier leakage, and thus reduce temperature sensitivity. ${ }^{9-12}$ The interface structure of InGaAs quantum wells on InGaAsP, integral to these improved structures, has however not been studied. In this letter, we report on the effects of substrate misorientation on the growth of strained-layer $\mathrm{In}_{0.18} \mathrm{Ga}_{0.82}$ As quantum wells with $\mathrm{In}_{0.29} \mathrm{Ga}_{0.71} \mathrm{As}_{0.4} \mathrm{P}_{0.6}$ (1.62 $\mathrm{eV}$ band gap) confinement layers and $\mathrm{InGaP}$ cladding layers lattice matched to a $\mathrm{GaAs}$ substrate. Low-temperature PL and AFM provide evidence of a strong dependence of interface structure on the substrate orientation, and correlates well with previously observed laser diode device performance. ${ }^{12}$

A schematic diagram of the InGaAs/InGaAsP/InGaP quantum well laser structure is shown in Fig. 1. These structures were grown by low-pressure (50 mbar) MOCVD in an Aixtron A-200 reactor at a growth temperature of $700{ }^{\circ} \mathrm{C}$. Trimethylgallium, trimethylindium, arsine, and phosphine

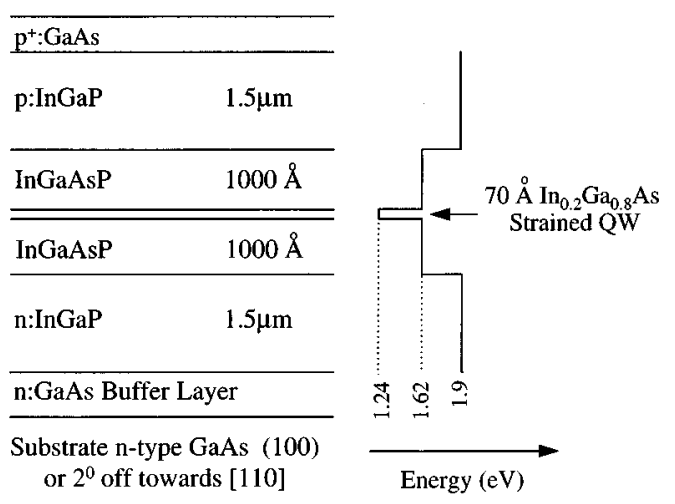

FIG. 1. Schematic cross section through laser structure. 


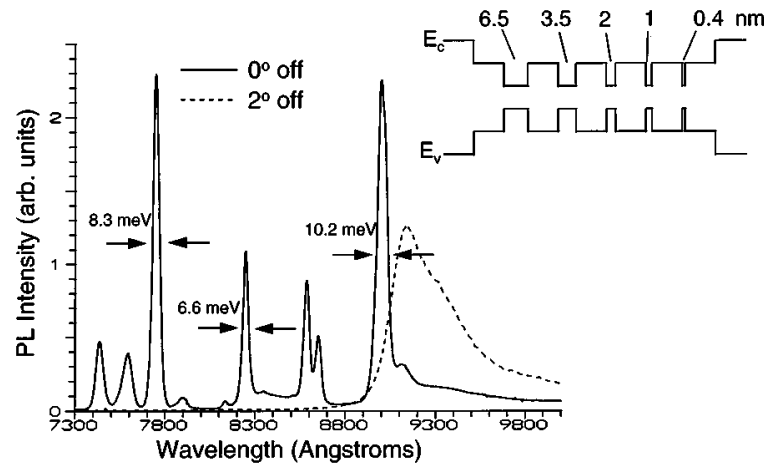

FIG. 2. $12 \mathrm{~K}$ photoluminescence spectra from an InGaAs/InGaAsP MQW structure grown on substrates exact $(100)$ and $2^{\circ}$-off towards [110].

were used as precursors in a $\mathrm{H}_{2}$ carrier-gas flow. The V/III ratios for the growth of InGaP, InGaAsP, and InGaAs layers were 200, 130, and 100, respectively. The growth rates, obtained from TEM micrographs, were $1.5 \mu \mathrm{m} / \mathrm{h}, 55 \mathrm{~nm} / \mathrm{min}$, and $15 \mathrm{~nm} / \mathrm{min}$ for InGaP, InGaAsP, and InGaAs layers, respectively, with the $\mathrm{InGaP}$ and InGaAsP layers having a lattice mismatch $\Delta a / a<0.001$ with respect to the GaAs substrate. The $n$-type GaAs substrates were 'epiready,' with no additional cleaning performed prior to growth. The interrupt times for switching between layers in the quantum wells were optimized to $2.5 \mathrm{~s}$ for the highest PL intensity. The AFM observations were made in air at room temperature.

The effect of substrate misorientation on the shape, intensity, and linewidth of the PL spectra at $12 \mathrm{~K}$ from quantum well thickness ranging from 0.5 to $6 \mathrm{~nm}$ has been studied. Cross-sectional TEM measurements are used to determine QW thickness. We have earlier reported ${ }^{13}$ on the substrate dependence of the $12 \mathrm{~K}$ PL spectra from a single InGaAs QW. Sharp multiplet emission, with a FWHM of 6-10 meV, is observed from exactly (100) substrates whereas signals from misoriented substrates $\left(2^{\circ}, 6^{\circ}, 10^{\circ}\right.$ off towards [110]) are weaker, much broader, and shifted to lower energies. Figure 2 shows PL from a multiquantum well structure with $5 \mathrm{InGaAs}$ wells. Narrow multiple-peak emission from the individual wells can be discerned only from the exact (100) substrate, whereas only a broad single peak is seen from the $2^{\circ}$-misoriented substrate. The multiple emission peaks observed here for on-orientation substrate growths are similar to features previously observed in the PL emission from InGaAs quantum wells on InP substrates. These spectral features have been attributed to a distribution in the variation in the quantum wells thickness, ${ }^{14}$ with the PL line shape being dependent on the length scale over which wellwidth fluctuations occur when compared to an excitonic diameter.

A series of samples were grown on (100) substrates, both exact, and $2^{\circ}$-misoriented towards [110], stopping at different layers in the laser structure in order to investigate the interfacial structure of the quantum-well laser material. AFM measurements were obtained for each of the surfaces that would represent interfaces during growth. Images of the surfaces of the epiready substrates, GaAs buffer layer, InGaP lower cladding, InGaAsP lower confinement, InGaAs quantum well, upper-confinement, and upper-cladding layers were thus obtained. While these micrographs were obtained $0^{\circ}-$ off

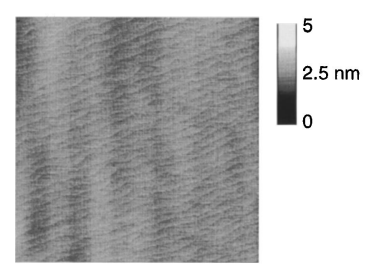

(b)

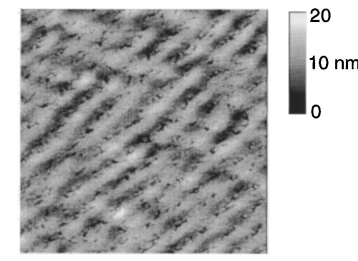

$2^{\circ}$ - off
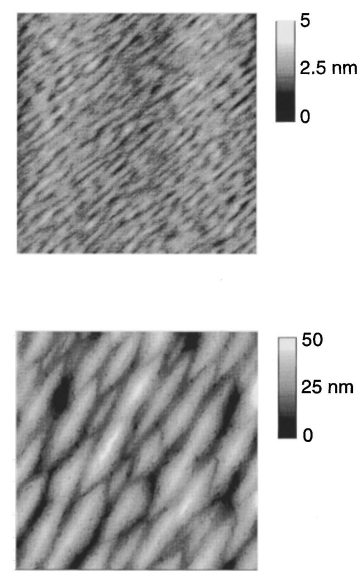

$10 \mu \mathrm{m}$
FIG. 3. AFM micrographs of (a) the GaAs buffer layer and (b) the $n:$ InGaP cladding layer grown on substrates exact $(100)$ and $2^{\circ}$-off towards [110].

after cooling in a stabilizing growth ambient, the cooling process is not expected to significantly alter the surface morphology. From these images, the evolution of the interface morphology during the growth of the laser structure can be discerned. The surface features of a given layer are dependent on the layer composition, as well as the cumulative roughness introduced by the underlying layers, and the substrate misorientation. The surfaces of the GaAs buffer layer [Fig. 3(a)] and the lower InGaP cladding layer [Fig. 3(b)] are smoother on the on-orientation substrates. The InGaP layer shows a terrace-type structure similar to that reported previously, ${ }^{15}$ with the terrace heights being higher on the $2^{\circ}$-off substrate. The surface roughness increases remarkably after the growth of the $100 \mathrm{~nm}$ quaternary confinement layer and the features on the InGaAs quantum well surface are markedly different for the $0^{\circ}$ - and $2^{\circ}$-off samples. AFM images of the confinement and quantum-well surfaces are shown in Fig. 4. Typical linescans through these images in the direction of the terraces are shown in Fig. 5. In both cases

(a)
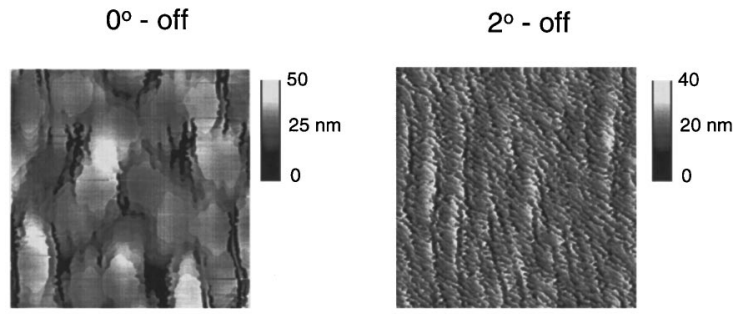

(b)
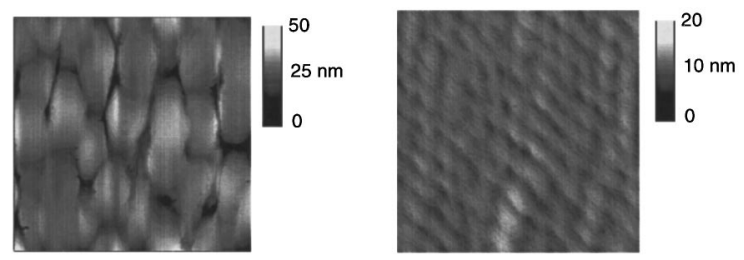

FIG. 4. AFM micrographs of the (a) InGaAs strained quantum well and (b) the lower InGaAsP confinement layer grown on substrates exact (100) and $2^{\circ}$-off towards [110]. 


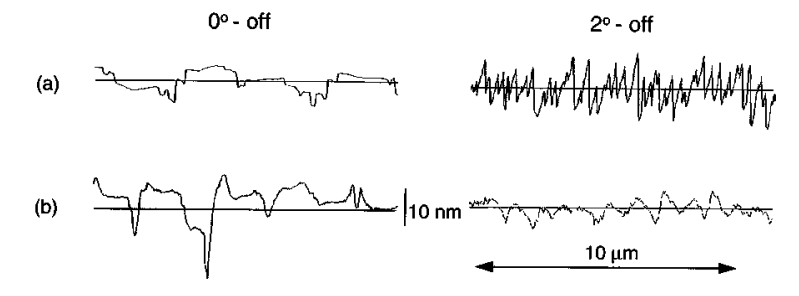

FIG. 5. Line scans through the AFM micrographs of Fig. 4 (a) InGaAs strained quantum well and (b) the lower InGaAsP confinement layer, taken in the direction of the terraces.

the interfacial roughness and structure is determined during the growth of the InGaAsP layer, the InGaAs quantum-well layer reproduces this underlying morphology. Large threedimensional island formation is observed for the InGaAsP layer on the exact (100) substrate; the subsequent InGaAs quantum-well layer grows conformally over structured surface. The islands themselves are a few microns in lateral extent and are locally smooth. The $2^{\circ}$-off samples possess a much rougher surface and hence interfaces. While the rms value of roughness of the InGaAs surface for both substrate orientations is approximately the same $(4.6 \mathrm{~nm})$, the $2^{\circ}$-off samples differ significantly in the lateral length scale of the roughness, which is only $0.2-0.3 \mu \mathrm{m}$.

The AFM measurements presented here allow for a direct correlation between the observed PL measurements and the interfacial structure of the heterointerfaces. Multiple PL peaks have been previously attributed ${ }^{14}$ to the presence of large smooth areas, that have lateral thickness variations larger than the excitonic diameter. Broad PL peaks have similarly been attributed to the presence of a substantial variation in QW thickness over an excitonic diameter. Our micrographs would indicate that the PL obtained from QW layers grown on exact (100) substrates should arise from the large flat plateaus found in Fig. 4. The spatial extent of these islands are typically $\approx 2 \mu \mathrm{m}$, which is large compared to an excitonic diameter. Multiple peaks arise from the slight variation in well thickness between the individual island regions. The roughness observed on the QW interfaces on the $2^{\circ}$-off substrates is on a much finer scale with no regions clearly exhibiting a planar morphology. This rougher morphology results in a greater variation in the local environment of the exciton and hence the luminescence represents an average of the wellwidths sampled, leading to a broader integrated signal. This broadening of PL linewidth from misoriented substrates has also been observed for InGaAs/GaAs quantum wells ${ }^{6}$ and has been attributed to step bunching during the growth on InGaAs on vicinal substrates.

Lasers grown on $2^{\circ}$-off substrates have been found to show an increased temperature sensitivity of the threshold current and differential quantum efficiency $\left(\eta_{d}\right)$ when compared with those grown on exact (100) substrates. ${ }^{13}$ For example, for 500-mm-long devices, the relative decrease in $\eta_{d}$ over the $20-55^{\circ} \mathrm{C}$ temperature range for lasers grown on $2^{\circ}$-off substrates is almost twice that of those grown on exact substrates. ${ }^{13}$ The characteristic temperature coefficients for both threshold $\left(T_{0}\right)$ and differential quantum efficiency $\left(T_{1}\right)$ are higher for devices grown on exact (100) substrates, which can be related to the interface morphology of the quantum-well active region. While the exact mechanism is not fully understood, the roughness of the QW interface on the misoriented substrate leads to an increased carrier leakage from the quantum well into the confinement layers, resulting in a lowering of the efficiency and increased threshold current with increasing temperature.

These studies have therefore directly shown that the performance of the Al-free laser structures is a strong function of the interfacial morphology resulting from a given substrate misorientation. Previous interpretation of PL spectra from QW structures based on a simple planar interface appears inadequate, and a detailed structural analysis, such as provided here, is necessary to understand device performance. The morphology of the active region is primarily determined by the morphology of the InGaAsP confinement layer, which is in turn impacted by substrate misorientation. Further improvements in the performance of these structures should be directly related to the growth conditions which can yield a planar morphology to the active region. The origin of the change in growth behavior, from a predominantly planar layer-by-layer growth, to an island or hillock structure, may be related to the proximity of the confinement-layer InGaAsP composition to the miscibility gap boundary in this material system. Optimization of these structures can therefore be carried out, in part, by the careful monitoring of the interface morphology of the active region.

${ }^{1}$ J. M. Olson, R. K. Ahrenkiel, D. J. Dunlavy, B. Keyes, and A. E. Kibbler, Appl. Phys. Lett. 55, 1208 (1989).

${ }^{2}$ J. Diaz, I. Eliashevich, K. Mobarhan, E. Kolev, L. J. Wang, D. Garbuzov, and M. Razeghi, IEEE Photon. Technol. Lett. 6, 132 (1994).

${ }^{3}$ W. Nakwaski, J. Appl. Phys. 64, 159 (1988).

${ }^{4}$ C. Zmudzinski, D. Botez, L. J. Mawst, A. Bhattacharya, M. Nesnidal, and R. F. Nabiev, IEEE J. Special Topics Quantum Electron. 1, 127 (1995).

${ }^{5}$ K. Ikuta, M. Shihonara, and N. Inoue, Jpn. J. Appl. Phys. 34, L220 (1995).

${ }^{6}$ K. Hiramoto, T. Tsuchiya, M. Sagawa, and K. Uomi, J. Cryst. Growth 145, 133 (1994).

${ }^{7}$ J. E. Epler, J. Soechtig, and H. C. Sigg, Appl. Phys. Lett. 65, 1949 (1994).

${ }^{8}$ E. C. Vail, R. F. Nabiev, and C. J. Chang-Hasnain, IEEE Photon. Technol. Lett. 6, 1303 (1994)

${ }^{9}$ M. Sagawa, T. Toyonaka, K. Hiramoto, K. Shinoda, and K. Uomi, Proceedings of 14th IEEE International Semiconductor Laser Conference, Sept. 19-23, Maui, Hawaii, p. 255, 1994.

${ }^{10}$ H. Asonen, A. Ovtchinnikov, G. Zhang, J. Nappi, P. Savolainen, and M. Pessa, IEEE J. Quantum Electron. 30, 415 (1994).

${ }^{11}$ M. Ohkubo, T. Ijichi, A. Iketani, and T. Kikuta, IEEE J. Quantum Electron. 30, 408 (1994)

${ }^{12}$ L. J. Mawst, A. Bhattacharya, M. Nesnidal, J. Lopez, D. Botez, J. A. Morris, and P. Zory, Electron. Lett. 31, 1153 (1995).

${ }^{13}$ L. J. Mawst, A. Bhattacharya, M. Nesnidal, J. Lopez, D. Botez, J. A. Morris, and P. Zory, Appl. Phys. Lett. 67, 2901 (1995).

${ }^{14}$ P. J. A. Phijs, PhD. thesis, Technical University, Delft, 1994.

${ }^{15}$ D. J. Friedman and G. S. Horner, Appl. Phys. Lett. 65, 878 (1994). 